Revue d'histoire de l'Amérique française

PGS REVUE D.HISTOIRE DE L'AMÉRIQUE FRANÇAISE

\title{
Les mouvements sociaux, la violence et les interventions armées au Québec 1867-1967
}

\section{Jean Pariseau}

Volume 37, numéro 1, juin 1983

URI : https://id.erudit.org/iderudit/304125ar

DOI : https://doi.org/10.7202/304125ar

Aller au sommaire du numéro

Éditeur(s)

Institut d'histoire de l'Amérique française

ISSN

0035-2357 (imprimé)

1492-1383 (numérique)

Découvrir la revue

Citer cet article

Pariseau, J. (1983). Les mouvements sociaux, la violence et les interventions armées au Québec 1867-1967. Revue d'histoire de l'Amérique française, 37(1), 67-79. https://doi.org/10.7202/304125ar d'utilisation que vous pouvez consulter en ligne.

https://apropos.erudit.org/fr/usagers/politique-dutilisation/ 


\title{
LES MOUVEMENTS SOCIAUX, LA VIOLENCE ET LES INTERVENTIONS ARMÉES AU QUÉBEC 1867-1967
}

\author{
JEAN PARISEAU \\ Défense Nationale, Ottawa
}

Malgré la preuve du contraire on continue à véhiculer la thèse de la non-violence dans le cours de l'histoire du Canada. Celle-ci fut inventée de toute part par les Loyalistes qui, fuyant la révolution américaine, cherchaient refuge au soi-disant «royaume paisible». Leurs historiens et ceux qui les suivirent se sont acharnés à faire croire que le républicanisme américain et la démocratie jacksonienne étaient imbus de violence - ce qui est amplement démontré par l'histoire - alors que le système parlementaire et monarchique britannique, dont le Canada a hérité, était synonyme du «bon ordre et de la paix» - ce qui ne concorde pas toujours avec la réalité historique'.

Nous avons déjà démontré ailleurs ${ }^{2}$ qu'au cours du siècle qui a précédé la Confédération canadienne, le pouvoir civil avait fait appel aux militaires pas moins de 250 fois en Grande-Bretagne, afin de réprimer des émeutes de toutes sortes, ce qui força éventuellement le gouvernement anglais à instituer un système policier efficace. Une situation semblable a eu lieu au Canada, alors que les troupes britanniques en garnison, rarement aidées des miliciens canadiens, sont intervenues une centaine de fois entre 1796 et 1866, soit la période de 70 ans qui a immédiatement précédé la Confédération.

Notre étude détaillée de 145 cas d'aide militaire au pouvoir civil au Canada, entre 1867 et 1967, nous a permis de bien situer le phénomène, surtout au niveau de la jurisprudence et des institutions en cause, notamment la police et l'armée. Comme en témoigne la liste suivante,

\footnotetext{
1 À titre d'exemples: William Kilbourn, ed., Canada, A Guide to the Peaceable Kingdom (Toronto, Macmillan, 1970); G.F.G. Stanley, Canada's Soldiers, 1604-1954: The Military History of an Unmilitary People (Toronto, Macmillan, 1954). Notre point de vue n'est pas original d'ailleurs; voir Kenneth McNaught, «Violence in Canadian History» dans J.S. Moir, ed., Character and Circumstances, Essays in honour of Donald Creighton (Toronto, U.T.P., 1970, 6684).

Voir J. Pariseau, «Forces armées et le maintien de i'ordre au Canada, 1867-1967: un siècle d'aide au pouvoir civil», thèse de D. ès L., Univ. Paul Valéry III, Montpellier (France), 1981 ( 5 tomes) (ci-après thèse).
} 
le tiers de ces interventions eut lieu au Québec où habitait en moyenne $30 \%$ de la population canadienne ${ }^{3}$.

\section{CAS D'AIDE AU POUVOIR CIVIL DANS LE QUÉBEC, 1867-1967}

APC

1. 1867- Grève, charpentiers de navires, QUÉBEC

2. " — Émeute appréhendée, élection, QUÉBEC

3. 1869- Grève, débardeurs, QUÉBEC

5. 1870 - Émeute appréhendée, QUÉBEC

6. " - Émeute appréhendée, élection, QUÉBEC

7. 1872- Émeute, élection, QUÉBEC

8. " - Émeute appréhendée, élection, MONTRÉAL

9. 1873- Émeute, élection, QUÉBEC

14. 1874 Émeute appréhendée, élection, QUÉBEC

15. " — Émeute appréhendée, élection, QUÉBEC et LÉVIS

20. ', - L'affaire Guibord, MONTRÉAL

25. 1877- Désordre, QUÉBEC

28. " - Agitation religieuse, MONTRÉAL

31. " - Perturbations appréhendées, voie ferrée, MANSONVILLE (Qué.)

32. " — Émeute appréhendée, élection, QUÉBEC

33. " - Émeute appréhendée, élection, QUÉBEC

34. " - Émeute, ouvriers du Canal Lachine, MONTRÉAL

35. 1878 - Émeute appréhendée, élection, QUÉBEC

36. " - Grève, ouvriers de la construction, QUÉBEC

37. " - Émeute appréhendée, défilé d'Orangistes, MONTRÉAL

40. " - Perturbations, construction du chemin de fer du nord, STETHÉRÈSE

41. " — Émeute appréhendée, élection fédérale, QUÉBEC

44. 1879- Émeute, débardeurs, QUÉBEC

47. 1880 - Émeute appréhendée, bateliers et ouvriers, QUÉBEC

48. 1881- Grève, débardeurs, MONTRÉAL

51. 1884- Perturbation, résistance à la construction d'une voie ferrée, AYLMER (Québec)

55. 1885- Émeute anti-vaccin, MONTRÉAL

56. 1886- Surveillance des terres de la Couronne, LÉVIS (Québec)

59. 1888- Perturbation, terrassiers, BURY et SAWYERVILLE (Québec)

62. 1891- Grève, employés de E.B. Eddy, HULL (Québec)

64. 1892- Surveillance des contrebandiers, ÎLE-AUX-COUDRES

66. 1895- Perturbation, refus de payer les taxes, BRENNAN'S HILL (Québec)

69. 1900 - Perturbation, étudiants des universités Laval et McGill, MONTRÉAL

72. ' - Grève, employés de la Dominion Cotton, MAGOG (Québec) 
73. " - Grève, employés de la Montreal Cotton, VALLEYFIELD (Québec)

75. 1903- Grève, débardeurs, MONTRÉAL

79. " - Perturbations au passage d'un cirque, ROBERVAL et GRAND-MÈRE (Québec)

82. 1906 - Grève, employés de Mac Laren Mills, BUCKINGHAM (Québec)

108. 1918 - Émeutes, crise de la conscription, QUÉBEC

112. " - Grève, policiers et pompiers, MONTRÉAL

116. 1919- Grève, ouvriers de la Davie Shipbuilding, LAUZON (Québec)

117. " - Grève, ouvriers de la Dominion Textile, MONTMORENCY (Québec)

118. 1921 - Grève, policiers et pompiers, QUÉBEC

133. 1941 - Grève, employés de la Foundation Company of Canada, ARVIDA (Québec)

135. 1943- Grève, employés de la société Aluminium of Canada, ARVIDA

138. ', - Grève, employés de la société Aluminium of Canada, SHAWINIGAN FALLS (Québec)

139. ' " - Grève, policiers et pompiers, MONTRÉAL

140. 1944 Grève, conducteurs de tramways, MONTRÉAL

145. 1966 - Menace de grève, Sûreté du Québec, PROVINCE DE QUÉBEC

\section{CAS D'ASSISTANCE AUX PÉNITENCIERS DANS LE QUÉBEC, 1867-1967}

PEN 8. 1962- Émeute, Pénitencier SAINT-VINCENT-DE-PAUL (Québec)

10. 1963- Émeute, Pénitencier SAINT-VINCENT-DE-PAUL (Québec)

À partir de cette liste on pourrait croire que les Québécois furent plus violents que la population canadienne en général, si l'on ne tenait pas compte du fait que Québec et Montréal étaient les villes commerciales les plus importantes du Canada au début des années étudiées. Ces deux villes ayant abrité des garnisons britanniques depuis la conquête ${ }^{4}$, les édiles étaient habitués à s'en remettre à celles-ci pour maintenir ou rétablir l'ordre lorsque leurs services policiers embryonnaires et mal organisés étaient insuffisants à la tâche ou tout simplement débordés ${ }^{5}$.

Il serait utile de réexaminer ce phénomène de l'intérieur, c'est-àdire à partir des mouvements sociaux, plutôt que de l'extérieur - du

4 Elinor Kyte Senior nous en donne un excellent exposé pour Montréal dans British Regulars in Montreal: An Imperial Garrison, 1832-1854 (Montréal, McGill-Queen's U.P., 1981).

5 À titre d'illustration on n'a qu'à se rappeler les émeutes Gavazzi à Québec et Montréal, en 1853, et le rapport des commissaires chargés d'enquêter sur la police à Québec à cette occasion. Voir P. Sylvain, Alessandro Gavazzi, clerc, Garibaldien et prédicant des Deux Mondes (Québec, Centre Pédagogique, (ca 1950); ibid., «Séjour mouvementé d'un révolutionnaire italien à Toronto et Québec», dans RHAF, XIII, 2: 173-216; et Report of the Commissioners appointed to inquire into the riot at Chalmers' Church, on the 6th of June, 1853, in Journals of the Legislative Assembly of the Province of Canada (Québec, 1855), 13, app. 7 (aussi donné en version française). 
point de vue institutionnel - comme nous l'avions fait précédemment. Rappelons qu'il ne s'agit pas ici d'une étude de la violence, ou d'une sociologie de la violence, mais seulement de cas de violence particuliers où sont intervenus les forces armées et des mouvements sociaux qui leur ont donné naissance.

Avant la Confédération, les gouvernements des provinces de l'Amérique du Nord britannique, à l'instar de l'Angleterre, promulguèrent des lois visant à maintenir l'ordre public. Au Canada, la responsabilité du maintien de l'ordre relève primordialement du système judiciaire et de la police, non de l'armée ${ }^{6}$, le rôle principal des forces armées étant de défendre l'État contre des agresseurs «externes». Mais advenant une situation où les policiers seraient débordés lors d'une émeute, ou risqueraient de l'être, le pouvoir civil, représenté jadis par les juges, les maires ou les juges de paix et, depuis 1924, par les procureurs généraux des provinces, a non seulement la responsabilité mais le devoir de faire appel aux militaires pour aider à maintenir ou à rétablir l'ordre public. Les militaires sont obligés par la loi de répondre à une telle réquisition ${ }^{7}$. Dans ces circonstances, ils sont nantis automatiquement des pouvoirs dévolus aux agents de la paix et sont donc fondés à employer la force raisonnablement nécessaire: "pour empêcher la perpétration d'une infraction (...) ou pour empêcher l'accomplissement de tout acte qui, à (leur) avis (...) constituerait une infraction (...).» ${ }^{8}$ Mais comme une loi de ce genre peut être sujette à diverses interprétations, on y trouve une clause freinante: «Quiconque est autorisé par la loi à employer la force est criminellement responsable de tout excès de force, selon la nature et la qualité de l'acte qui constitue l'excès.» ${ }^{9}$ À l'encontre de l'opinion publique, qui croit que les militaires ont tous les droits dans de telles circonstances, on s'aperçoit, au contraire, qu'ils sont assujettis à la loi tout comme les civils eux-mêmes, en plus d'être soumis à des règlements militaires sévères. Ceux-ci rappellent aux militaires qu'ils ne remplacent pas l'autorité civile mais l'aident à maintenir le respect du droit et de l'ordre; qu'il incombe au représentant du pouvoir civil de demander au commandant des troupes en présence «d'entrer en action»; que ce dernier doit «examiner si une action immédiate s'impose»; doit «prendre les mesures qu'il juge nécessaires»; qu'il a «lorsqu'il est convaincu que la situation rend impérative l'emploi d'armes à feu (...) un pouvoir discrétionnaire à l'égard de tout ordre donné aux officiers et aux hommes placés sous son commandement au sujet de l'ouverture du

\footnotetext{
6 Code criminel, art. 2, agent de la paix; pour une étude détaillée du rôle des policiers, voir: W. et N. Kelly, Policing in Canada (Toronto, Macmillan, 1976), 704 p. On en trouve aussi des résumés dans Bovey, op. cit., 248-257; J. Ricker et J. Saywell, How we are governed (Toronto, Clarke, Irwin \& Co.) 144-151.

Loi sur la Défense nationale, partie XI, révisée en 1972. Pour un historique, voir J. Pariseau, thèse, I: chap. I.

Code criminel, art. $2 \mathrm{f}$ et 27.

9 Ibid., art. 26.
} 
feu (...)»; enfin qu'il doit «arrêter toute action entreprise quand il lui apparaît qu'il n'est plus nécessaire de la poursuivre». De plus, aucun officier ni homme n'ouvre le feu sauf sur l'ordre d'un officier de commandement supérieur ou immédiat ou d'un officier désigné par l'un d'eux, et il «doit le faire de telle sorte qu'il puisse cesser le feu dès qu'il n'est plus nécessaire ${ }^{10}{ }$. On voit que ces règlements respectent en tous points les dispositions du Code criminel quant à l'emploi de la force et ses limitations. Et bien que ces citations fassent partie des règlements récents elles sont essentiellement semblables à ceux en vigueur depuis la Confédération.

Comme on ne saurait étudier ici tous les mouvements sociaux du Québec, nous nous bornerons à en étudier deux en particulier - l'antimilitarisme et le sectarisme religieux. Nous regarderons de près deux aspects: la violence et les interventions armées au Québec.

\section{Anti-militarisme}

Les «émeutes de la conscription» qui ont suivi la mise en application de la loi du Service militaire, dans la ville de Québec, en mars/ avril 1918, ont souvent été interprétées comme un cas patent d'antimilitarisme québécois ${ }^{11}$. Pourtant les Canadiens français avaient raison de détester la guerre ou du moins le service militaire, si l'on tient compte des conditions de service qui leur étaient imposées à la Première Guerre mondiale. Un rappel historique nous aidera à mieux comprendre cette situation.

Durant le Régime français les Canadiens n'ont cessé de démontrer leur courage au cours de deux guerres franco-iroquoises et de quatre guerres coloniales franco-anglaises. La défaite de l'armée régulière française sur les hauteurs d'Abraham et les terribles déprédations exercées sur les propriétés canadiennes par les réguliers britanniques créèrent, chez les Canadiens, une aversion pour la guerre et pour le service militaire. Et si les Québécois ont hésité, en 1775, à se défendre contre des envahisseurs, c'est parce que ceux-ci étaient soutenus par la France et que secrètement on espérait que le Canada serait repris aux Anglais. Pourtant, en 1812 la situation n'était plus du tout la même, de sorte que les Canadiens français n'ont pas hésité à défendre leur territoire contre les Américains; il en fut de même en 1866-1870, contre les Féniens.

Les soulèvements de 1837-1838 sont d'excellents exemples d'antimilitarisme, soit du côté des Patriotes où l'on remarque un dégoût prononcé pour la discipline militaire, soit du côté du reste de la population

10 Ordonnance et Règlements royaux applicables aux Forces canadiennes, I (Administration): alinéas 23.03, 23.13,23.14, 23.15. À noter que l'emploi de la baïonnette, non mentionnée dans les règlements, est considérée comme une mesure moins aléatoire que celle de l'emploi d'armes à feu.

11 Consulter la bibliographie exhaustive dans J. Pariseau, op. cit., 4, APC 108: 35-43. 
qui refuse son soutien aux rebelles. Par ailleurs, en 1868, la réponse des Zouaves donnerait-elle à entendre que l'esprit guerrier se réveille lorsque les Québécois s'identifient à la cause du Souverain Pontife? Rappelons simplement que bon nombre d'entre eux étaient des cadets ou des miliciens qui se virent confier le rôle d'instructeurs, une fois rendus en Italie ${ }^{12}$. Une situation inverse a existé pendant la Guerre sudafricaine justement parce que les Québécois considéraient ce conflit comme injuste envers les Boers alors que la majorité de l'élite urbaine anglo-canadienne épousait la cause «jingoïste» britannique de plainpied.

Mais tous ces conflits ne faisaient que préfigurer l'attitude adoptée par la majorité des Québécois au cours de la Première Guerre mondiale. L'usage de la langue française, tant au sein de la milice permanente que de la milice non-permanente, n'était pas reconnu. Il existait bien quatre unités d'artillerie et une quinzaine de bataillons canadiens-français ${ }^{13}$, mais l'instruction et l'exercice s'y faisaient presque entièrement en anglais, de même que toute la correspondance officielle. Un seul officier supérieur du grade de colonel avait servi à l'état-major avant d'être mis au rancart par le nouveau ministre de la Milice et de la Défense, l'honorable Sam Hughes, en $1911^{14}$. Au début des hostilités, ce dernier ne tient pas compte du plan de mobilisation et crée le Corps expéditionnaire canadien (C.E.C.). C'est à son corps défendant qu'il accepte, en fin de compte, qu'une seule unité francophone, le $22^{\mathrm{e}}$ Bataillon canadien-français, combatte au sein du Corps d'Armée canadien en France ${ }^{15}$. Cette attitude, soutenue par le Gouvernement conservateur jusqu'à la démission forcée de Sam Hughes, le 9 novembre 1916, et renforcée par l'acharnement des Anglo-Ontariens à vouloir supprimer les écoles françaises en Ontario, n'a certainement pas aidé à favoriser une plus grande participation des Canadiens français à la Grande Guerre ${ }^{16}$. Et l'imposition tardive de la conscription a eu l'effet de détourner à tout jamais la confiance des Québécois envers le parti conservateur fédéral.

12 R. Hardy, Les zouaves (Montréal, Boréal Express, 1980), 152. L. Lamontagne, «Habits gris et chemise rouge», dans CHAR (1950): 20-29.

${ }_{13}$ En plus de quatre batteries d'artillerie on trouve 15 bataillons d'infanterie, soit le $4^{\mathrm{e}}$ Chasseurs canadiens, le $9^{\mathrm{e}}$ Voltigeurs de Québec, le $17^{\mathrm{e}}$ Régt (Lévis), le $18^{\mathrm{e}}$ Régt Franc-tireurs du Saguenay, le 54 $4^{\mathrm{e}}$ Régt Carabiniers de Sherbrooke, le $61^{\mathrm{e}}$ Régt de Montmagny, le 64 Régt de Châteauguay-Beauharnois, le $65^{\mathrm{e}}$ Carabiniers Mont-Royal, le $80^{\mathrm{e}}$ Régt de Nicolet, le $83^{\mathrm{e}}$ Régt de Joliette, le $84^{\mathrm{e}}$ Régt de Ste-Hyacinthe, le $85^{\mathrm{e}}$ Régt (Montréal), le $86^{\mathrm{e}}$ Régt de Trois-Rivières, le $87^{\mathrm{e}}$ Régt de Québec, le $89^{\mathrm{e}}$ Régt de Témiscouata et Rimouski et le $92^{\mathrm{e}}$ Régt de Dorchester: Militia List 1913 (Ottawa, M.D.N.).

14 Voir J. Pariseau, «Étude No 1: Le bilinguisme et le biculturalisme au ministère de la Défense, 1946-1963», MS (Ottawa, Service historique de la Défense, 1980), 5.

15 J.-P. Gagnon, «Le 22e Bataillon (canadien-français), 1914-1919. Une étude sociale», MS provisoire (Ottawa, Service historique de la Défense, 1981), $1^{\text {er }}$ chap: 15 et seq.

16 Mason Wade, The French Canadians, 1760-1945 (Toronto, Macmillan, 1955), 608 à 780; J.L. Granatstein et J.M. Hitsman, Broken Promises, A History of Conscription in Canada (Toronto Oxford U.P., 1977), 22-104. 
De fait, l'anti-militarisme donna lieu à de violentes manifestations à Montréal en 1916 et en $1917^{17}$, mais c'est dans la ville de Québec, pendant la Semaine Sainte de 1918, que se révéla l'ampleur du sentiment anti-militaire canadien-français. À la suite d'émeutes au cours desquelles le public saccagea les bureaux du préposé au Service militaire, l'armée fut appelée à intervenir, à $22 \mathrm{~h}$. le Vendredi Saint, 29 mars, par le maire qui avait déjà trop tergiversé ${ }^{18}$. Le brigadier-général J.-L. Landry, commandant le district militaire No 5 (Québec), refusa la réquisition parce qu'elle ne portait que la signature du maire au lieu de celle de trois juges de paix, tel que prescrit par la loi. Entre-temps, après en avoir averti le G.Q.G. de la Milice à Ottawa, il intervint avec 800 hommes. L'ordre fut rétabli à 22 h 30 sans que les soldats aient eu recours à leurs armes. Le lendemain matin, 30 mars, le général Landry demanda des renforts au G.Q.G., sur quoi le Conseil de la Milice fit envoyer 1200 conscrits anglophones de Toronto, qu'il plaça sous les ordres du major général François-Louis Lessard. Le dimanche de Pâques se passa sans incident notable, mais une émeute éclata à nouveau le lundi soir, $\mathrm{e}^{\mathrm{er}}$ avril, après que des constables spéciaux de la Dominion Police eurent tenté d'arrêter de jeunes réfractaires à la loi du service militaire. Des émeutiers ouvrirent le feu contre les militaires; en retour, la troupe répondit avec les armes, tuant quatre civils, et en blessant plusieurs. Quelque 70 personnes furent mises aux arrêts, une fois l'ordre rétabli.

Le 4 avril, le Gouverneur en conseil, par voie d'un arrêté en conseil rétroactif au $1^{\mathrm{er}}$ avril, déclara opérante la loi martiale dans la région de Québec ${ }^{19}$. Les émeutes cessèrent mais l'attitude anti-militaire persista jusqu'à la fin de la guerre. «Aucun événement n'a divisé les Canadiens de façon si marquée que la question de conscription pour service militaire outre-mer en temps de guerre», dira l'historien J.L. Granatstein $^{20}$. Cette crise fut sans aucun doute la plus sérieuse des crises nationales, outre la grève générale de Winnipeg en 1919 et la crise d'octobre en 1970.

Pendant la Guerre civile d'Espagne, en 1936, c'est peut-être autant par anti-militarisme que parce qu'ils favorisaient le parti catholique représenté par Franco, que les Canadiens français ne se sont pas joints aux mercenaires.

\footnotetext{
17 Les 29 et 30 août 1917 , un homme fut tué et 4 policiers blessés lors d'une manifestation anti-conscriptioniste, M. Wade, The French Canadians, 747.

18 Voir J. Pariseau, thèse, monographie APC 108. Dans cette étude et plus particulièrement dans un compte rendu paru dans Livres et Auteurs québécois 1971, nous expliquons notre désaccord avec l'interprétation de Jean Provencher, Québec sous la loi des mesures de guerre, 1918 (Montréal, Boréal Express, 1971).

19 J. Pariseau, 4, APC 108.

20 J.L. Granatstein et J.M. Hitsman, Broken Promises, A History of Conscription in Canada (Toronto, Oxford U.P., 1977), 1.
} 
Au cours de la Deuxième Guerre mondiale, nous retrouvons encore des relents d'anti-militarisme, quoique moins profonds et moins répandus. Les Canadiens français ont trouvé une certaine ouverture d'esprit dans le gouvernement libéral, qui n'existait pas chez les conservateurs. Bien que la marine et l'aviation soient des contreforts unilingues anglais, l'armée de terre, elle, reconnaît la valeur des soldats francophones. Il est vrai que l'absence de cours techniques dans les institutions d'enseignement du Québec réduit les chances de servir dans les branches qui exigent ces qualifications. Mais on retrouve au moins quatre bataillons d'infanterie, un régiment d'artillerie et plusieurs unités ancillaires, où le français est parlé et où on se sent plus à l'aise ${ }^{21}$. Les manifestations anti-militaires ne manquent pas: Asbestos (1942), Portneuf et Montréal (1943), Montréal et Rimouski (1944), et Drummondville (1945) sont parmi les plus bruyantes, mais elles ne ressemblent en rien aux émeutes de 1918 à Québec. La police militaire montre toujours autant de zèle à traquer les déserteurs mais on sent que les politiciens ne veulent pas répéter l'erreur de 1918. Pourtant les conservateurs, qui ne sont plus au pouvoir, font mousser l'opinion publique chez les anglophones et, par le truchement d'un plébiscite, forcent le gouvernement à établir la conscription pour le service outre-mer. Au même moment, fait inconnu de la grande majorité des Canadiens, l'Aviation royale du Canada regorge de candidats, si bien qu'on devra en laisser partir quelque 40000 à l'automne de 1944. Ce seul geste, s'il avait été posé au printemps, aurait suffi à désamorcer la crise «artificielle» des renforts ${ }^{22}$ et, du coup, à diminuer l'anti-militarisme au Québec.

On peut donc conclure, de cet exposé sommaire, que l'anti-militarisme a été tantôt latent, tantôt virulent au Québec. Il n'a pas empêché une participation active, quoique numériquement limitée, des Québécois à certaines guerres. Une seule confrontation majeure de nature antimilitaire a nécessité une intervention armée durant le premier centenaire de la Confédération, ce qui démontre le peu de violence physique suscité par le mouvement anti-militariste au Québec. La contre-violence de l'armée, par contre, imposée largement par suite de l'inaction des policiers, causa quatre morts et plusieurs blessés. Pourtant, lorsqu'on étudie froidement l'action militaire, on découvre que celle-ci fut effectuée avec modération si on la compare à d'autres incidents semblables, plus récents, aux Indes et en Afrique du sud, par exemple. Elle n'en demeure pas moins un acte honteux dans notre histoire, qui aurait pu

\footnotetext{
21 Jean-Yves Gravel, «Le Québec militaire, 1939-1945», dans ibid., Le Québec et la guerre (Montréal, Boréal Express, 1974), 77-108.

22 W.A.B. Douglas et B. Greenhous, Out of the Shadows: Canada in the Second World War (Toronto, Oxford U.P., 1977), 246-247. La question de la main-d'oeuvre est étudiée à fond dans Mag.-gén. E.L.M. Burns, Manpower in The Canadian Army 1939-1945 (Toronto, Clarke, Irwin \& Co. Ltd., 1956), 184 p., sauf pour la question du surplus d'aviateurs, qui sera étudiée dans le $2^{\mathrm{e}}$ tome de l'histoire officielle de l'A.R.C. (Ottawa, Service historique du Q.G.D.N.), à paraître $c a 1984$.
} 
être évité avec un peu de bonne volonté de la part d'un gouvernement décidé à réprimer des sujets récalcitrants.

\section{Sectarisme religieux}

Avant 1867, la violence accompagnant l'implantation des loges orangistes dans la province du Canada suscita plusieurs interventions militaires. Nous en avons relevé huit cas entre 1824 et $1866^{23}$, auxquels il faut ajouter l'émeute Gavazzi à Québec en $1853^{24}$.

La Confédération ne mit pas fin aux conflits sectaires, semble-til, puisque ceux-ci continuèrent au-delà du siècle. On compte de nombreux affrontements entre les membres de l'Institut canadien ou entre les orangistes et les catholiques; mais trois seulement furent l'objet d'interventions militaires au Québec.

La première eut lieu le 16 novembre 1875 , lors de l'inhumation de Joseph Guibord au cimetière catholique de la Côte-des-Neiges, à Montréal. Mort six ans et demi plus tôt, Guibord n'avait pu recevoir la sépulture en lieu consacré parce qu'il avait été membre de l'Institut canadien. L'affaire fut traduite en cour, et après la cassation d'un premier jugement par la Cour d'Appel du Québec et la confirmation de celui-ci par la Cour du Banc de la Reine, l'Institut fit appel au comité judiciaire du Conseil privé de Londres et gagna sa cause. C'est donc lors d'une seconde tentative d'inhumation - une première ayant échoué à cause des menaces de la foule - que la milice intervint, s'interposant entre le cortège funèbre et la foule excitée ${ }^{25}$. Il n'y eut aucune contreviolence, et l'Affaire Guibord fut consignée aux livres d'histoire.

Deux ans plus tard, la population de Montréal était à nouveau surexcitée à l'approche de la célébration annuelle de la bataille de la Boyne. Le matin du 12 juillet 1877, le défilé des Orangistes fut contremandé après que les autorités municipales eurent refusé toute protection spéciale. Mais lorsqu'un jeune orangiste du nom de Hackett fut tué au cours de l'après-midi, le commandant du district militaire fit appel à la «Brigade de Montréal» pour mettre fin à l'émeute, ce qui se fit sans recours aux armes.

Les obsèques de Hackett, fixées au mardi 17 juillet, permirent à quelque 3000 Orangistes de l'extérieur d'y assister. Venant surtout des Cantons de l'Est et de l'Ontario, ils arrivèrent nombreux dans le but de gonfler le cortège funèbre, en témoignage de sympathie. Le maire

\footnotetext{
${ }^{23}$ Ces cas sont tirés surtout de: H. Senior, Orangeism: The Canadian Phase (Toronto, McGraw-Hill Ryerson, 1973), passim.

24 «Report of the Commissioners appointed to enquire into the conduct of the police authorities on the occasion of the riot at Chalmers' Church on the 6th of June, 1853, dans Journals of the Legislative Assembly of the Province of Canada (1855), 13: app. 7. Voir aussi P. Sylvain, Allessandro Gavazzi, clerc, Garibaldien et prédicant des Deux Mondes (Québec, Centre pédagogique, $c a$ 1950), et l'article dans la RHAF, XIV: 173-216.

25 J. Pariseau, thèse, monographie, APC 20.
} 
Beaudry crut jusqu'au dernier moment que son corps policier municipal suffirait à maintenir l'ordre, mais devant un tel attroupement et la possibilité de sérieux affrontements, il signa, avec d'autres juges de paix, une réquisition d'aide militaire au pouvoir civil. Tout le long de la procession les troupes s'interposèrent de chaque côté, entre celle-ci et les spectateurs, depuis la cathédrale anglicane jusqu'au cimetière MontRoyal, de sorte qu'il n'y eut aucune contre-violence ${ }^{26}$.

L'année suivante, l'effervescence reprit à l'approche du 12 juillet. Cette fois, appuyée par une loi fédérale prohibant l'emploi d'armes à feu et par un nombre impressionnant de constables spéciaux, le maire Beaudry fit arrêter les chefs orangistes dès qu'ils tentèrent de mettre le défilé en marche ${ }^{27}$. Cet acte mit fin au désir des Orangistes de tenter une épreuve de force; du coup les 3000 miliciens canadiens, appelés en service à l'aide du pouvoir civil et commandés par le général Edward Selby-Smith, furent renvoyés chez eux sans avoir eu à affronter les émeutiers.

L'affaire Guibord fut surtout le résultat d'une querelle d'idées, menée dans les journaux, plutôt qu'une démonstration de violence physique; on y décèle un conflit entre le dogmatisme des ultramontains et le libéralisme des membres de l'Institut canadien. La violence collective survenue lors des défilés orangistes, par contre, fut le résultat d'un affrontement voulu, planifié et exécuté par une société irlandaise créée peu après la bataille de la Boyne en 1690, dans le but de favoriser l'essor du protestantisme, de la monarchie, de l'empire britannique, et prônant le rejet du «papisme» ${ }^{28}$; on n'aurait pu inventer une société qui allait plus à l'encontre des idéologies et des intérêts des Irlandais catholiques et des Canadiens français. Importée au Canada d'abord par les soldats réguliers britanniques, et établie par Ogle Robert Gowan vers 1830, elle visait surtout l'entraide mutuelle des immigrants irlandais et comptait déjà plusieurs milliers d'adhérents avant la Confédération. L'historien G.S. Kealey, qui a relevé pas moins de 22 émeutes ou perturbations causées par les Orangistes dans la seule ville de Toronto, de 1867 à 1892, suppose que la violence était «ritualisée» et prenait l'une des formes suivantes: les catholiques attaquent les Orangistes lors des défilés annuels ou autres démonstrations; les Orangistes essaient d'imposer leur présence en «territoire catholique», ce qui porte les catholiques à rétorquer. L'affrontement de Montréal fut le pire du genre, en termes de violence, mais la volonté des autorités civiles fédérales,

26 Ibid., APC 28.

27 Ibid., APC 37 . Il s'agit bien de la «loi pour la meilleure prévention des crimes violents» du Parlement du Canada (41 Vict., c. 17, promulguée le 10 mai 1878), tel que cité dans Peter B. Waite, Canada 1874-1896, Arduous Destiny (Toronto, McClelland \& Stewart, 1971), 88 et non du statut de la Province de Québec (41-42 Vict., c. 9) sanctionné seulement le 20 juillet 1878, donc huit jours après l'émeute. $13-14$

H. Senior, «The Genesis of Canadian Orangeism», dans Ontario History (juin 1968): 
provinciales et municipales, de même que celle des autorités militaires, à vouloir maintenir l'ordre, fut telle que les Orangistes cessèrent leurs démonstrations publiques majeures après 1878 pour adopter la clandestinité. On voit justement ce changement d'attitude lorsqu'on compare deux autres incidents d'aide militaire au pouvoir civil: l'émeute de Caraquet (N.-B.) en 1875 et celle de Ford City (Windsor, Ont.) en 1917. Le premier révèle un affrontement public de la part des autorités locales anglo-protestantes contre des parents franco-catholiques qui ne cherchent qu'à éduquer leurs enfants selon leurs croyances religieuses et dans leur langue maternelle. Le second, par contre, est le résultat d'une action concertée entre les fonctionnaires orangistes du gouvernement ontarien et monseigneur Fallon, évêque francophobe de London (Ont.), qui ne reconnaissaient pas aux francophones le droit de tenir des écoles dans leur langue ${ }^{29}$. Dans les deux cas l'ordre fut rétabli par les policiers avant même l'entrée en fonction des militaires.

Que peut-on conclure sur les mouvements sociaux et la violence à partir des exemples cités?

L'anti-militarisme québécois semble avoir été lié assez intimement au nationalisme canadien-français. Très souvent latent, ce phénomène ressurgissait à l'occasion des guerres. D'après les journaux de l'époque, on distingue difficilement entre les excès de pacifisme et d'anti-militarisme. On ne hait pas les querelles de mots, on les recherche même. Le Québécois n'hésite pas à défendre son territoire contre l'envahisseur américain ou fénien; il n'hésite pas non plus à accourir à la défense du pape, son père spirituel, lorsque celui-ci est menacé. Mais même si ses ancêtres ont accepté la Conquête, il ne ressent aucun lien, aucun attachement particulier à la Couronne d'Angleterre, encore moins au gouvernement impérialiste. Depuis la Révolution française, il a aussi modifié ses sentiments envers la France athée et les Français républicains. Ainsi, il considère injuste qu'on exige de lui qu'il aille combattre les Boers dans une guerre impérialiste qui favorise les intérêts capitalistes anglais; il considère injuste qu'on lui demande de verser son sang pour la France et l'Angleterre dont les seules ambitions semblent être la domination du plus puissant sur les moins bien nantis. Et il va jusqu'à fomenter une série d'émeutes pour illustrer son opposition aux manigances d'un gouvernement conservateur qui ne lui reconnaît même pas le droit de servir sa patrie dans sa langue maternelle. Cette violence est, de fait, de la contre-violence, puisqu'elle est la seule réponse qu'il sache donner aux constables spéciaux de la Dominion Police - anglophones en grande majorité - qui ont recours à toutes les ruses possibles pour dépister et arrêter les récalcitrants alors qu'eux-mêmes esquivent le service militaire outre-mer. On verra des séquelles de cette même

29 J. Pariseau, thèse, APC 17 et 107. 
attitude durant la Deuxième Guerre mondiale, quoique moins prononcées.

Pendant longtemps les historiens ont rarement mentionné ou ont minimisé l'existence de mouvements semblables au Canada anglais. Ce n'est que récemment, dans une étude fouillée sur le recrutement pendant la Grande Guerre, qu'on rapporte en détail les attitudes des anglophones ${ }^{30}$.

Le sectarisme religieux, d'autre part, était inévitable dans le cours de notre histoire. La conquête du Canada avait laissé environ 60000 Canadiens français catholiques sous la domination d'Anglo-protestants. Il était «normal» pour ces derniers de tenter de faire triompher le protestantisme comme ils l'avaient fait au Pays de Galles, en Écosse et en Irlande. Il faut admettre, pourtant, que le Canada n'a pas connu la violence des razzias de Cromwell. Et si, au début, l'ostentation des Orangistes visait plutôt les Irlandais catholiques, elle n'a pas manqué d'entraîner l'antipathie des Canadiens français. Par contre, il était tout aussi prévisible que le libéralisme et l'ultramontanisme qui «opposa farouchement l'Eglise (catholique) aux libéraux européens et sud-américains, ne fut pas moins violente au Canada français» ${ }^{31}$. Mais ici aussi, la violence se fit surtout dans les journaux. Les quelques affrontements physiques furent surtout rituels et aucune des interventions militaires n'entraîna de sérieuses conséquences.

Certains historiens ont minimisé l'état de la violence au Canada dans le but de justifier le mythe qui veut que «l'ordre et le bon gouvernement», rejeté par l'esprit révolutionnaire et républicain, soit l'apanage du système monarchique et parlementaire britannique dont le Canada a hérité. D'autres ont amplifié les querelles et la violence au point de faire croire que le Québec ne pourrait jamais s'auto-gouverner à moins d'une révolution ou d'une séparation. Cette étude nous a permis de constater que le Québec a connu des moments de violence, animés par des mouvements sociaux distincts mais semblables à ceux des autres provinces, durant le centenaire qui a suivi la Confédération. Si nous avons pu déceler certains aspects de la spécificité québécoise, nous devons reconnaitre que le Québec, tout différent qu'il soit des autres provinces, a connu ses mouvements sociaux propres et a encouru ses moments de violence tout comme les autres provinces mais ni plus,

\footnotetext{
30 Robert Craig Brown et Donald Loveridge, «Unrequited Faith: Recruiting the CEF 19141918» dans Revue internationale d'Histoire militaire, no 51 (Édition canadienne) Ottawa, Commission canadienne d'Histoire militaire, 1982: 53-79. Le professeur Desmond Morton dans l'article liminaire de cette revue «The Military Problems of an Unmilitary Power» (p. 10) rappelle que de nombreux Anglophones des provinces maritimes et de l'Ontario, dont les souches canadiennes étaient fermement ancrées, s'étaient montrés tout autant isolationnistes et anti-militaires que les Québécois durant la Guerre des Boers.

P. Sylvain, «Libéralisme et ultramontanisme au Canada français: affrontement idéologique et doctrinal (1840-1865)», dans W.L. Morton, éd., Le Bouclier d'Achille (Montréal, McClelland \& Stewart, 1968), 111.
} 
ni moins. Pourtant c'est au Québec qu'on retrouve les seuls cas d'intervention militaire où des personnes furent tuées ${ }^{32}$ durant cette même période. Hasard ou préméditation? On ne saurait affirmer ni l'un ni l'autre.

Enfin, qu'on me permette de rappeler que dans 145 cas d'aide au pouvoir civil et dans 11 cas d'assistance dans les pénitenciers, entre 1867 et 1967, au Canada, les forces armées, par leur seule présence, ont réussi à maintenir ou rétablir l'ordre sans avoir recours à la contreviolence, dans $92 \%$ des cas. Devant une évidence si frappante, il est temps de démolir certains mythes historiques bien établis au Canada.

\footnotetext{
32 En plus des 4 hommes tués et des quelques blessés lors des émeutes anti-conscriptionnistes à Québec, en 1918, un homme avait été tué et quelques-uns blessés, lors d'une grève des ouvriers de la construction en 1878. En 1962, les détenus du pénitencier de Saint-Vincent-de-Paul de Montréal se soulevèrent, causant $\$ 3$ millions de dommages aux immeubles. Les militaires furent appelés. Un prisonnier fut tué et 26 blessés par les forces de l'ordre avant que l'ordre ne soit rétabli. Voir J. Pariseau, thèse, APC 36 et 108 et PEN 8.
} 